\title{
Strange Attractors in Periodically-kicked Limit Cycles and Hopf Bifurcations
}

\author{
Qiudong Wang ${ }^{1}$ and Lai-Sang Young ${ }^{2}$
}

\begin{abstract}
We prove the emergence of chaotic behavior in the form of horseshoes and strange attractors with SRB measures when certain simple dynamical systems are kicked at periodic time intervals. The settings considered include limit cycles and stationary points undergoing Hopf bifurcations.
\end{abstract}

This paper is about a mechanism for producing chaos. The scheme consists of periodic kicks interspersed with long periods of relaxation. We apply it to some very tame dynamical settings, such as limit cycles and stable equilibria undergoing Hopf bifurcations, and prove the appearance of chaotic behavior under reasonable conditions.

The results in this paper, beginning with the statements in Section 1, are rigorous. The rest of this introduction is devoted to a nontechnical discussion of some of the ideas and issues surrounding this work.

\section{Main results}

In Theorem 1, we prove that when suitably kicked, all limit cycles can be turned into strange attractors with strong stochastic properties.

Theorems 2 and 3 have to do with Hopf bifurcations. In the absence of forcing, the picture for a supercritical Hopf bifurcation is classical and well known: a stable fixed point loses its stability when a pair of complex conjugate eigenvalues crosses the imaginary axis, resulting in the appearance of a limit cycle which increases in diameter as it moves away from the newly unstable fixed point. Subjecting this system to periodic kicks, we prove that if there is a sufficiently strong "twist" at the fixed point and the forcing is of a suitable type, then in lieu of the limit cycle, a strange attractor sometimes emerges from the bifurcation.

The appearance of horseshoes is also proved in both situations.

The results above are related as follows. For arbitrary limit cycles, even though the geometric principles are clear, it is difficult to formulate a quantitative statement without detailed knowledge of the local geometry. In Hopf bifurcations, this information is contained in the first three derivatives at the bifurcating point. It can be condensed, in fact, into a single number called the "twist factor".

\section{Horseshoes and strange attractors}

We clarify what is meant by "horseshoes" and "strange attractors".

"Horseshoes" as introduced by Smale [S] are invariant Cantor sets on which the map is hyperbolic and has positive topological entropy. Since they are not attracting, these sets represent, from the observational point of view, transient chaos.

\footnotetext{
${ }^{1}$ Dept. of Math., University of Arizona, Tucson, AZ 85721, email dwang@math.arizona.edu. This research is partially supported by a grant from the NSF

${ }^{2}$ Courant Institute of Mathematical Sciences, 251 Mercer St., New York, NY 10012, email lsy@cims.nyu.edu. This research is partially supported by a grant from the NSF
} 
The term "strange attractor" is used in this paper to summarize a number of precisely defined dynamical properties that together imply sustained, observable chaos. These properties include, for example, positive Lyapunov exponents starting from almost all initial conditions in the basin, statistical coherence in the sense of orbits in large sets organizing themselves according certain special invariant measures called SRB measures, and strong stochastic properties such as exponential correlation decay for sequences of observations of the type $\varphi, \varphi \circ F, \cdots, \varphi \circ F^{n}, \cdots$. (See Sect. 1.1 for more detail.)

Horseshoes are created by "stretch-and-fold" type actions. They are robust; once they develop, they persist. The creation of strange attractors with SRB measures requires a balance that is considerably more subtle. For the logistic family, it has been proved that two kinds of maps with opposite behaviors - those with invariant densities and those with periodic sinks - partition parameter space in a complicated way; see e.g. [L]. There is evidence of a similar picture for invertible maps in $2 \mathrm{D}$; see e.g. [N], [WY2]. Though yet unsubstantiated, current thinking is that outside of the Axiom A category, SRB measures in general is a "positive probability phenomenon" rather than one that occurs on open sets of parameter space, even when the map has stretch-and-fold geometry.

Our results in Theorems 1-3 reinforce this emerging picture: Our a priori conditions for the existence of strange attractors are more stringent than those for horseshoes. When these conditions are met, we prove the presence of horseshoes for open sets of parameters, and strange attractors for maps corresponding to parameters in a positive measure set.

\section{A mechanism for the production of chaos}

Our scheme relies on the natural forces of shear to exaggerate the irregularities brought on (deliberately) by the kick. We explain - on an intuitive level - how this works in Hopf bifurcations in $2 \mathrm{D}$.

For argument's sake, let 0 be the fixed point and $\Gamma=\{r=\sqrt{\mu}\}$ be the emerging limit cycle, $\mu$ being the bifurcation parameter. Suppose we give the system a kick in the radial direction, distorting the shape of $\Gamma$ as shown in Fig. 1(a). Generically, the unforced system has a "twist", meaning points at different distances from 0 rotate at different speeds. During the relaxation period, some points on this distorted circle rotate ahead of others. A stretch-and-fold action results if the twist is sufficiently strong; see Fig. 1(b).

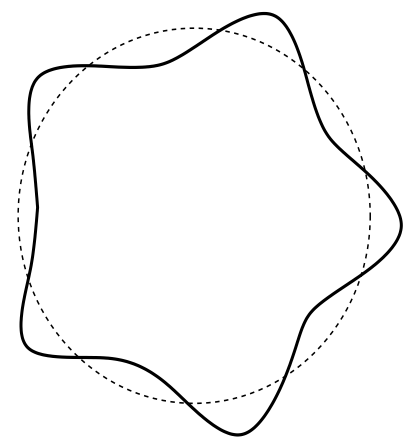

(a)

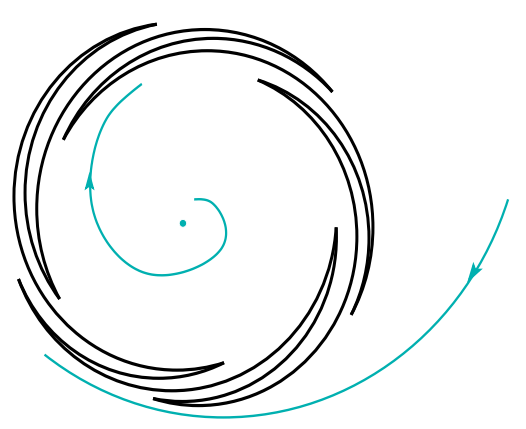

(b)

Figure 1 A Hopf attractor 


\section{Supporting analysis}

We focus on strange attractors as the corresponding proofs for horseshoes are quite simple. Our analysis is based on [WY1] and [WY3], which together contain one of the very few rigorous theories of strange attractors besides Axiom A theory. We discuss briefly below the approach in these two papers and how to apply the results.

[WY1] and [WY3] are about maps with attracting sets on which there is strong dissipation and (in most places) a single direction of instability. Two-parameter families $\left\{T_{a, b}\right\}$ are considered. Roughly speaking, $a$ is a parameter that allows us to effect changes along the unstable direction and $b$ is the inverse of "dissipation". The idea is to try to pass to the singular limit at $b=0$ to obtain a one-paramter family of $1 \mathrm{D}$ maps. Now 1-dimensional objects are considerably simpler than $n$-dimensional objects, and the theory of $1 \mathrm{D}$ maps is fairly well developed. We proved in [WY1] and [WY3] that if the singular limit above makes sense, and if the resulting family of 1D maps has certain good properties, then some of these properties can be passed back to $b>0$, and they in turn allow us to prove the desired results on strange attractors for a positive measure set of $a$.

Parts of the analysis in [WY1] and [WY3] have their origins in [BC], which contains the pioneering work on Hénon attractors. Some of the ideas in $[\mathrm{BC}]$ in turn come from the theory of $1 \mathrm{D}$ maps; see $[\mathrm{J}]$ in particular.

We emphasize that in contrast to earlier results, the theory in [WY1] and [WY3] is "generic", in the sense that the conditions under which it holds pertain only to certain general characteristics of the maps and not to specific formulas or contexts. To prove Theorems 1-3, we will show that the present setting fits into the framework of [WY1] and [WY3], and then leverage the results in these two papers.

\section{Relation to previous works}

An application of $[\mathrm{BC}]$ to show the presence of strange attractors (in a weaker sense than explained above) in 2D homoclinic bifurcations is given in [MV].

The first evidence of chaotic behavior in periodically forced systems goes back to the work of Cartwright and Littlewood on the van der Pol oscillator [CL]. Levinson [Ln] made considerable progress on a simplified (linearized) model of this system, for which thirty years later Levi [Li] proved the existence of horseshoes. Numerical work by Zaslavsky [Z] suggested the presence of strange attractors for an even simpler second order equation. Not knowing about $[\mathrm{Z}]$, we studied again the same equation recently and proved (rigorously) the existence of horseshoes and strange attractors [WY2].

To our knowledge, the forcing of limit cycles had not been studied previously in the generality of Theorem 1 of this paper. Also, to our knowledge, chaotic behavior in connection with Hopf bifurcations had not been observed or predicted prior to this work.

We mention also a connection of a different kind. Not all of our perturbations are small; large kicks are sometimes needed to "break" limit cycles. But under suitable circumstances, such as when the twist factor in a Hopf bifurcation is large, we prove that strange attractors may result from mild disturbances applied at infrequent time intervals. In this regard our results are in the direction of Ruelle and Takens [RT]; see also [NRT]. Our mechanism, which relies on shear, is different and perhaps more natural. 


\section{Precise Statements of Results}

\subsection{Horseshoes and strange attractors}

In this subsection we isolate and define precisely a number of dynamical properties commonly associated with chaos. These are the properties which will appear in our results stated in Sects. 1.2-1.4.

The following is a slight generalization of Smale's horseshoe as introduced in [S]:

(i) Let $f: M \rightarrow M$ be a $C^{1}$ embedding of a Riemannian manifold $M$ into itself with a compact invariant set $\Lambda$. We say $\left.f\right|_{\Lambda}$ is uniformly hyperbolic if there is a continuous splitting of the tangent bundle over $\Lambda$ into $E^{u} \oplus E^{s}$ such that $\left.D f\right|_{E^{u}}$ is expanding and $\left.D f\right|_{E^{s}}$ is contracting (i.e. $\exists C>0$ and $\lambda>1$ s.t. for all $v \in E^{u},\left\|D f^{n}(v)\right\| \geq$ $C \lambda^{n}\|v\| \forall n \geq 0$ etc.)

(ii) Let $\Sigma_{n}=\{1,2, \cdots, n\}^{\mathbb{Z}}$, and let $\sigma: \Sigma_{n} \rightarrow \Sigma_{n}$ be the shift operator. Then $\left(\Sigma_{n}, \sigma\right)$ is called the full shift on $n$ symbols.

An embedding $f: M \rightarrow M$ is said to have a horseshoe if

(H) for some $N, n \in \mathbb{Z}^{+}, f^{N}$ has a uniformly hyperbolic invariant set $\Lambda \subset M$ such that $\left.f^{N}\right|_{\Lambda}$ is topologically conjugate to $\left(\Sigma_{n}, \sigma\right)$.

Horseshoes are robust in the sense that they persist under perturbations of $f$. Having a horseshoe is a notion of topological chaos. It implies in particular that $f$ has positive topological entropy. But since horseshoes usually have Lebesgue measure zero, it is entirely possible for a map to have a horseshoe and at the same time to have the orbit of Lebesguealmost every point tend to a stable equilibrium.

Next we describe a dynamical picture which is chaotic not only from the topological but also from the probabilistic point of view; it represents a stronger form of chaos. Let $f$ be an embedding such that $f(\bar{U}) \subset U$ for some open set $U$. In this paper, we refer to $\Omega:=\cap_{n \geq 0} f^{n}(\bar{U})$ as an attractor and $U$ as its basin. Our dynamical results include a number of properties frequently associated with "strange attractors". (We regard the term "strange attractor", which embodies a wide range of ideas, as descriptive rather than technically defined.) We give precise definitions of the relevant properties below, and label them as (SA1)-(SA4). Later on, to remind the reader what (SA1)-(SA4) stands for, we will refer to an attractor $\left.f\right|_{\Omega}$ having these properties as "a strange attractor with (SA1)-(SA4)".

First, we recall the definition of SRB measures. An invariant Borel probability measure $\nu$ for $f$ is called an SRB measure if $f$ has a positive Lyapunov exponent $\nu$-almost everywhere and the conditional measures of $\nu$ on unstable manifolds are equivalent to the Riemannian volume on these leaves. See $[\mathrm{Y}]$ for more information.

The following are properties we associate with the idea of strange attractors:

(SA1) Positive Lyapunov exponents For Lebesgue-a.e. $x \in U$, the orbit of $x$ has a positive Lyapunov exponent, i.e.

$$
\lim _{n \rightarrow \infty} \frac{1}{n} \log \left\|D f^{n}(x)\right\|>0 .
$$


(This property is important enough that we state it separately; it, in fact, follows from (SA2).)

(SA2) Existence of SRB measures and basin property

(a) $f$ admits at least one and at most finitely many ergodic SRB measures all of which have no zero Lyapunov exponents; we denote them by $\nu_{1}, \cdots, \nu_{r}$;

(b) for Lebesgue-a.e. $x \in U, \exists j=j(x)$ such that for every continuous function $\varphi: U \rightarrow \mathbb{R}$

$$
\frac{1}{n} \sum_{i=0}^{n-1} \varphi\left(f^{i} x\right) \rightarrow \int \varphi d \nu_{j} .
$$

\section{(SA3) Statistical properties of dynamical observations}

(a) For every ergodic SRB measure $\nu$ and every Hölder continuous function $\varphi: \Omega \rightarrow \mathbb{R}$, the sequence $\left\{\varphi \circ f^{i}\right\}_{i=0,1, \cdots}$ obeys a Central Limit Theorem, i.e. if $\int \varphi d \nu=0$, then $\frac{1}{\sqrt{n}} \sum_{0}^{n-1} \varphi \circ f^{i}$ converges in distribution to the normal distribution, and the variance is strictly positive unless $\varphi \circ f=\psi \circ f-\psi$ for some $\psi$.

(b) Suppose that for some $N \geq 1, f^{N}$ has an $S R B$ measure $\nu$ that is mixing. Then given a Hölder exponent $\eta, \exists \tau=\tau(\eta)<1$ such that for all $\varphi, \psi: \Omega \rightarrow \mathbb{R}$ Hölder with exponent $\eta, \exists K=K(\varphi, \psi)$ such that $\forall n \geq 1$,

$$
\left|\int\left(\varphi \circ f^{n N}\right) \psi d \nu-\int \varphi d \nu \int \psi d \nu\right| \leq K(\varphi, \psi) \tau^{n}
$$

We remark that all ergodic SRB measures with no zero Lyapunov exponents are mixing up to a finite factor.

The first part of (SA2) can sometimes be strengthened to

\section{(SA4) Uniqueness of SRB measure and ergodic properties}

(a) $f$ admits a unique (and hence ergodic) SRB measure $\nu$;

(b) $(f, \nu)$ is mixing or, equivalently, isomorphic to a Bernoulli shift.

\subsection{Chaotic behavior in periodically kicked limit cycles}

A periodic orbit $\gamma$ of a flow is called a limit cycle if it is attractive, a hyperbolic limit cycle if the eigenvalues of its section maps have moduli $<1$. It is well known that hyperbolic limit cycles are robust, so that given a flow $\varphi_{t}$ with such a cycle and a time $T$, if $\kappa$ is a small perturbation, then under the iteration of $\varphi_{T} \circ \kappa$, all points continue to be attracted to a simple closed curve. Theorem 1 describes some possible scenarios if larger perturbations are permitted.

Theorem 1 (Creation of strange attractors from limit cycles) Let $\varphi_{t}$ be a $C^{4}$ flow on an $n$-dimensional Riemannian manifold $M$. Assume that $\varphi_{t}$ has a hyperbolic limit cycle $\gamma$. For $n=2$, assume also that the normal bundle to $\gamma$ is orientable. Let $U$ be a 
neighborhood of $\gamma$, and let $\operatorname{Emb}^{3}(U, M)$ be the space of $C^{3}$ embeddings of $U$ into $M$. Then there is an open set $\mathcal{E} \subset \mathrm{Emb}^{3}(U, M)$ such that the following hold for every $\kappa \in \mathcal{E}$ :

(i) $\varphi_{T} \circ \kappa$ has a horseshoe (i.e. Property $(\mathbf{H})$ ) for all large $T$;

(ii) $\varphi_{T} \circ \kappa$ has a strange attractor with (SA1)-(SA4) for a positive measure set of $T$.

Remark The ideas embodied in Theorem 1 can be expressed in many different ways. The presence of a parameter is important, for as we will see in Section 2, our conclusion will invariably be that maps corresponding to a positive measure set of parameters have strange attractors. In Theorem 1, we have chosen - for convenience - this parameter to be $T$, the time interval between consecutive kicks. One can, for example, fix $T$ and use instead one or more parameter families of kick maps with suitable properties. We leave these variants of our results to the reader, our intent here being only to illustrate the general idea.

\subsection{Periodically forced Hopf bifurcations in 2D}

The following are the general equations for a Hopf bifurcation in 2D, written in normal form in complex coordinates:

$$
\dot{z}=\lambda_{\mu} z+a_{\mu} z^{2} \bar{z}+b_{\mu} z^{3} \bar{z}^{2}+\cdots
$$

where $\mu \in \mathbb{R}$ is a parameter, $a_{\mu}, b_{\mu} \in \mathbb{C}$ are constants, and $\lambda_{\mu}=\mu+\left(\omega+\mu \gamma_{\mu}\right) i, \gamma_{\mu}, \omega \in \mathbb{R}$, $\omega \neq 0$. That is to say, at the bifurcation parameter $\mu=0$, the linearized equation at the bifurcation point $z=0$ is $\dot{z}=i \omega z$, and that $\lambda_{\mu}$ crossses the imaginary axis from left to right as $\mu$ increases past zero.

For our purposes, it is convenient to write these equations in polar coordinates:

$$
\begin{aligned}
& \dot{r}=\left(\mu-\alpha_{\mu} r^{2}\right) r+r^{5} g_{\mu}(r, \theta), \\
& \dot{\theta}=\omega+\gamma_{\mu} \mu+\beta_{\mu} r^{2}+r^{4} h_{\mu}(r, \theta) .
\end{aligned}
$$

Here $\omega, \alpha_{\mu}, \beta_{\mu}, \gamma_{\mu} \in \mathbb{R}$ are constants, and $g_{\mu}$ and $h_{\mu}$ are functions which we will assume to be of class $C^{4}$; all depend smoothly on $\mu$. ( $\omega$ and $\gamma$ are as in equation (1), while $a=-\alpha+i \beta$.) In addition to assuming $\omega \neq 0$, we consider in this paper the case $\alpha>0$, i.e. the case of a generic supercritical Hopf bifurcation. From the main terms of equation (2), one sees that as $\mu$ increases from 0 , an attracting invariant circle of radius $\approx \sqrt{\frac{\mu}{\alpha}}$ appears.

For references on Hopf bifurcations, see e.g. [H], [GH] and [MM].

From here on we omit the subscript $\mu$ in the constants in our equations except where the dependence on $\mu$ is at issue.

To the system described by equation (2), we give a kick which leaves 0 fixed and which is radial in space and periodic in time, resulting in

$$
\begin{aligned}
& \dot{r}=\left(\mu-\alpha r^{2}\right) r+r^{5} g(r, \theta)+r \Phi(\theta) \sum_{n=0}^{\infty} \delta(t-n T), \\
& \dot{\theta}=\omega+\gamma \mu+\beta r^{2}+r^{4} h(r, \theta) .
\end{aligned}
$$

Here $\delta(\cdot)$ is the usual $\delta$-function, $\Phi: S^{1} \rightarrow \mathbb{R}$ is a $C^{3}$ function, and $T$ is the period of the kick. Equation (3) is to be interpreted as follows: Since the kicks are radial, they do 
not affect the $\theta$-coordinate. At times $t=n T, n=0,1,2, \cdots$, the $r$-coordinate changes abruptly from $r_{-}(n T)$ to $r_{+}(n T)$ where $r_{+}(n T):=\lim _{\varepsilon \rightarrow 0} r_{\varepsilon}(\varepsilon)$ and $r_{\varepsilon}(t)$ is the solution of $\dot{r}_{\varepsilon}=\frac{1}{\varepsilon} r_{\varepsilon} \Phi(\theta)$ with $r_{\varepsilon}(0)=r_{-}(n T)$, that is to say,

$$
r_{+}(n T)=r_{-}(n T) e^{\Phi(\theta(n T))} .
$$

During the time interval $(n T,(n+1) T)$, the system evolves according to $(2)$ with initial position $\left(r_{+}(n T), \theta(n T)\right)$ and final position $\left(r_{-}((n+1) T), \theta((n+1) T)\right)$. For each $\mu$, let $F_{\mu, T}: \mathbb{R}^{2} \rightarrow \mathbb{R}^{2}$ denote the time- $T$-map of the system defined by (3).

Theorem 2 Assume $\frac{\beta_{0}}{\alpha_{0}} \neq 0$, and let $\Phi_{0}: S^{1} \rightarrow \mathbb{R}$ be a $C^{3}$ function with nondegenerate critical points. We consider forcing functions $\Phi$ of the form $\Phi=A \Phi_{0}, A \in(0, \infty)$. In (1) and (2) below, we assert that if $\left|\frac{\beta_{0}}{\alpha_{0}}\right| A$ is sufficiently large, then there is a set of parameters $(\mu, T)$ for which $F_{\mu, T}$ has chaotic behavior.

(1) (Existence of horseshoes) There exists $K_{0}=K_{0}\left(\Phi_{0}\right)$ such that if $\left|\frac{\beta_{0}}{\alpha_{0}}\right| A>K_{0}$, then for all $\mu$ in some interval $\left(0, \mu_{0}\right)$, there exist $T_{0}(\mu)=\mathcal{O}\left(\frac{1}{\mu}\right)$ and an open set $\Delta_{0}(\mu) \subset\left(T_{0}, \infty\right), \Delta_{0}(\mu)$ roughly $\frac{2 \pi}{\omega}$-periodic, such that for all $T \in \Delta_{0}(\mu), F_{\mu, T}$ has a horseshoe, i.e. Property $(\mathbf{H})$. If $\left|\frac{\beta_{0}}{\alpha_{0}}\right| A$ is sufficiently large, then $\Delta_{0}(\mu)=\left(T_{0}, \infty\right)$.

(2) (Existence of strange attractors) There exists $K_{1}=K_{1}\left(\Phi_{0}\right)$ such that if $\left|\frac{\beta_{0}}{\alpha_{0}}\right| A>$ $K_{1}$, then the following hold for all $\mu$ in some interval $\left(0, \mu_{1}\right)$ : There exist $T_{1}(\mu)=\mathcal{O}\left(\frac{1}{\mu}\right)$ and a positive Lebesgue measure set $\Delta_{1}(\mu) \subset\left(T_{1}, \infty\right), \Delta_{1}(\mu)$ roughly $\frac{2 \pi}{\omega}$-periodic, such that for all $T \in \Delta_{1}(\mu), F_{\mu, T}$ has a strange attractor with (SA1)-(SA3). If $\left|\frac{\beta_{0}}{\alpha_{0}}\right| A$ is sufficiently large, then $F_{\mu, T}$ has property (SA4) as well.

In general, the larger the twist factor $\left|\frac{\beta_{0}}{\alpha_{0}}\right|$, the weaker the forcing required. If $\left|\frac{\beta_{0}}{\alpha_{0}}\right|>>1$, then very mild disturbances (with e.g. $\Phi(\theta)=\varepsilon \sin \theta$ ) at periodic time intervals can give rise to strange attractors. Also, $T_{1}$ is usually $>>T_{0}$.

Remark Our purpose here is to bring to light the phenomenon of chaos appearing in periodically-kicked Hopf bifurcations, and to call the reader's attention to a relevant set of mathematical tools. No attempt has been made to formulate the most general results. In Theorems 2 and 3, for example, kicks that are not radial can be considered. To gain more insight into what kind of kicks are suitable, see the intuitive explanation in the introduction. For alternate formulations regarding parameters, see the Remark following Theorem 1.

\subsection{Hopf bifurcations in higher dimensions}

The following is a direct generalization of our results in 2D: Consider as before a 1-parameter family of equations on $\mathbb{R}^{n}$ having a stationary solution at 0 . Suppose that for $\mu<0$, the eigenvalues at 0 all have strictly negative real parts, and that at $\mu=0$, a pair of complex conjugate eigenvalues crosses the imaginary axis. We decompose the tangent space at 0 into $\mathbb{R}^{n}=V^{c} \oplus V^{s}$ where $V^{c}$ is the 2-dimensional subspace corresponding to the leading pair of eigenvalues and $V^{s}$ is the invariant subspace corresponding to the rest. The existence 
of a center manifold $W^{c}$ tangent to $V^{c}$ is well known. We assume that the bifurcation is supercritical, and that the unforced equation restricted to $W^{c}$ is in normal form. (See Sect. 4.5 for the precise meaning of this last condition.)

To this bifurcation, we add a forcing that is radial. Let $S^{n-1}=\left\{u \in \mathbb{R}^{n}:\|u\|=1\right\}$, $\bar{\Phi}_{0}: S^{n-1} \rightarrow \mathbb{R}$, and $\bar{u}=\frac{u}{\|u\|}$. We consider a forcing of the form $A \bar{\Phi}_{0}(\bar{u}) u \sum_{n=0}^{\infty} \delta(t-n T)$.

Theorem 3 Let $\alpha$ and $\beta$ be as in equation (2) for the unforced equation restricted to $W^{c}$, and assume $\left.\bar{\Phi}_{0}\right|_{\left(S^{n-1} \cap V^{c}\right)}$ is $C^{3}$ with nondegenerate critical points. Then results analogous to those in Theorems 2 hold.

\section{Sufficient Conditions for Strange Attractors (and Horse- shoes)}

In this section we recall some results on the existence of strange attractors of certain types. The framework here is considerably more general than that in Section 1; it is unrelated to Hopf bifurcations or limit cycles. We will focus primarily on conditions that guarantee (SA1)-(SA4). Weaker conditions that guarantee the presence of horseshoes are discussed in Sect. 2.3.

\subsection{Conditions for (SA1)-(SA4)}

We recall in this subsection some earlier results which will be used in this paper. [WY1], which contains a $2 \mathrm{D}$ version of the results below, is the backbone of the strange attractor results in all of our theorems. A couple of useful modifications of the conditions in [WY1] are pointed out in [WY2]. The $n$-dimensional version of [WY1] is proved in [WY3]. This version is used in the proofs of Theorems 1 and 3.

We consider a family of maps $T_{a, b}: M=S^{1} \times D \rightarrow M$ where $D$ is the closed unit disk in $\mathbb{R}^{n-1}, a \in\left[a_{0}, a_{1}\right] \subset \mathbb{R}$ and $b \in B_{0}$ where $B_{0}$ is any subset of $\mathbb{R} \backslash\{0\}$ with an accumulation point at $0 .^{3}$ Points in $M$ are denoted by $(x, y)$ with $x \in S^{1}$ and $y \in D$.

Very roughly, the parameters $a$ and $b$ can be thought of as having the following meanings: a moves points along $S^{1} \times\{0\}$, which, in most places, is a direction of instability, while $\frac{1}{|b|}$ can be interpreted as a measure of dissipation. In particular, $T_{a, 0}$ has infinite dissipation; it sends all of $M$ to a one-dimensional object (see (C1) below).

\section{(C0) Regularity conditions}

(i) For each $b \in B_{0}$, the function $(x, y, a) \mapsto T_{a, b}(x, y)$ is $C^{3}$;

(ii) each $T_{a, b}$ is an embedding of $M$ into itself;

(iii) there exists $K>0$ independent of $(a, b)$ such that for all $(a, b)$,

$$
\frac{\left|\operatorname{det} D T_{a, b}(z)\right|}{\left|\operatorname{det} D T_{a, b}\left(z^{\prime}\right)\right|} \leq K \quad \forall z, z^{\prime} \in S^{1} \times D
$$

\footnotetext{
${ }^{3}$ The formulation here (with $b \neq 0$ ) together with $(\mathbf{C 1})$ is equivalent to that in [WY1] and [WY3]. We have elected to state $(\mathbf{C 1})$ as a separate condition because in applications, the definition of $T_{a, b}$ for $b \neq 0$ usually comes for free while the existence of the singular limit $T_{a, 0}$ has to be proved.
} 


\section{(C1) Existence of singular limit}

There exist $T_{a, 0}: M \rightarrow S^{1} \times\{0\}, a \in\left[a_{0}, a_{1}\right]$, such that as $b \rightarrow 0$, the maps $(x, y, a) \mapsto T_{a, b}(x, y)$ converge in the $C^{3}$ norm to $(x, y, a) \mapsto T_{a, 0}(x, y)$.

Identifying $S^{1} \times\{0\}$ with $S^{1}$, we refer to $T_{a, 0}$ as well as its restriction to $S^{1} \times\{0\}$, i.e. the family of $1 \mathrm{D}$ maps $f_{a}: S^{1} \rightarrow S^{1}$ defined by $f_{a}(x)=T_{a, 0}(x, 0)$, as the singular limit of $T_{a, b}$. The rest of our conditions are imposed on the singular limit alone.

The next condition in [WY1] or [WY3] is the existence of $a^{*} \in\left[a_{0}, a_{1}\right]$ such that $f=f_{a^{*}}$ satisfies the so-called Misiurewicz condition. In practice, we have found that (C2) below is more directly checkable, albeit a little more cumbersome to state. That (C2) implies the condition in [WY1] and [WY3] is proved in [WY2], Appendix A.

\section{(C2) Existence of a sufficiently expanding map from which to perturb}

There exists $a^{*} \in\left[a_{0}, a_{1}\right]$ such that $f=f_{a^{*}}$ has the following properties: There are numbers $c_{1}>0, N_{1} \in \mathbb{Z}^{+}$, and a neighborhood $I$ of the set $C:=\left\{f^{\prime}=0\right\}$ such that

(i) $f$ is expanding on $S^{1} \backslash I$ in the following sense:

(a) if $x, f x, \cdots, f^{n-1} x \notin I, n \geq N_{1}$, then $\left|\left(f^{n}\right)^{\prime} x\right| \geq e^{c_{1} n}$;

(b) if $x, f x, \cdots, f^{n-1} x \notin I$ and $f^{n} x \in I$, any $n$, then $\left|\left(f^{n}\right)^{\prime} x\right| \geq e^{c_{1} n}$;

(ii) $f^{n} x \notin I \quad \forall x \in C$ and $n>0$;

(iii) in $I$, the derivative is controlled as follows:

(a) $\left|f^{\prime \prime}\right|$ is bounded away from 0 ;

(b) by following the critical orbit, every $x \in I \backslash C$ is guaranteed a recovery time $n(x) \geq 1$ with the property that $f^{j} x \notin I$ for $0<j<n(x)$ and $\left|\left(f^{n(x)}\right)^{\prime} x\right| \geq e^{c_{1} n(x)}$.

Next we introduce the notion of smooth continuations. Let $C_{a}$ denote the critical set of $f_{a}$. For $x=x\left(a^{*}\right) \in C_{a^{*}}$, the continuation $x(a)$ of $x$ to $a$ near $a^{*}$ is the unique critical point of $f_{a}$ near $x$. If $p$ is a hyperbolic periodic point of $f_{a^{*}}$, then $p(a)$ is the unique periodic point of $f_{a}$ near $p$ having the same period. It is a fact that in general, if $p$ is a point whose

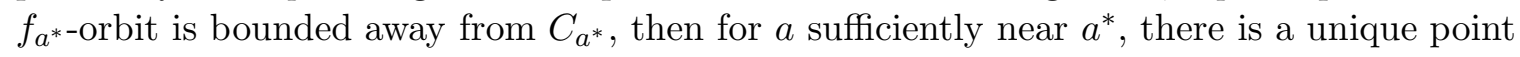
$p(a)$ with the same symbolic itinerary under $f_{a}$.

\section{(C3) Parameter transversality}

For each $x \in C_{a^{*}}$, let $p=f(x)$, and let $x(a)$ and $p(a)$ denote the continuations of $x$ and $p$ respectively. Then

$$
\frac{d}{d a} f_{a}(x(a)) \neq \frac{d}{d a} p(a) \quad \text { at } a=a^{*} .
$$

(C4) Nondegeneracy at "turns"

$$
\frac{\partial}{\partial y} T_{a^{*}, 0}(x, 0) \neq 0 \quad \forall x \in C_{a^{*}}
$$




\section{(C5) Conditions for mixing}

(i) $e^{c_{1}}>2$ where $c_{1}$ is in (C2).

(ii) Let $J_{1}, \cdots, J_{r}$ be the intervals of monotonicity of $f_{a^{*}}$, and let $P=\left(p_{i, j}\right)$ be the matrix defined by

$$
p_{i, j}=\left\{\begin{array}{c}
1 \quad \text { if } f\left(J_{i}\right) \supset J_{j}, \\
0 \text { otherwise }
\end{array}\right.
$$

Then there exists $N_{2}>0$ such that $P^{N_{2}}>0$.

Theorem A ([WY1], [WY3]) Suppose $\left\{T_{a, b}\right\}$ satisfies conditions $(\mathbf{C 0})-(\mathbf{C 4})$. Then for all sufficiently small $b \in B_{0}$, there is a positive measure set of a for which $T_{a, b}$ has properties (SA1), (SA2) and (SA3).

Theorem B ([WY1], [WY3]; and appendix of [WY2]) In the sense of Theorem A, $(\mathrm{C0})-(\mathrm{C} 5) \Longrightarrow(\mathrm{SA} 1)-(\mathrm{SA} 4)$.

\subsection{Model singular limit maps}

In this subsection, we consider an (abstractly defined) class of 1D maps which satisfy Conditions (C2), (C3) and (C5) in Sect. 2.1. The maps in this class will be shown later on to arise as singular limits in the situations of interest.

Proposition 2.1 Let $\Phi: S^{1} \rightarrow \mathbb{R}$ be a $C^{3}$ function with nondegenerate critical points. Then there exist $L_{1}$ and $\delta$ depending on $\Phi$ such that if $L \geq L_{1}$ and $\Psi: S^{1} \rightarrow \mathbb{R}$ is a $C^{3}$ function with $\|\Psi\|_{C^{2}} \leq \delta$ and $\|\Psi\|_{C^{3}} \leq 1$, then the family

$$
f_{a}(\theta)=\theta+a+L(\Phi(\theta)+\Psi(\theta)), \quad a \in[0,1],
$$

satisfies (C2) and (C3) in Sect. 2.1. (C5) holds if $L_{1}$ is sufficiently large.

This is a slightly more general setting than that treated in Sects. 5.2 and 5.3 of [WY2], but the proofs are identical.

\subsection{Conditions for horseshoes}

It is a general fact that the existence of an SRB measure with nonzero Lyapunov exponents implies the presence of horseshoes. This follows from

Theorem $[\mathrm{K}]$ Let $f: M \rightarrow M$ be a $C^{1+\alpha}$ diffeomorphism of a compact Riemannian manifold, and let $\mu$ be an invariant probability measure with (i) nozero Lyapunov exponents and $\left(\right.$ ii) $h_{\mu}(f)>0$. Then $f$ has a horseshoe.

In general, horseshoes appear considerably before strange attractors. We give a sufficient condition in the spirit of Sect. 2.1. The following applies to one value of $a$ at a time, so let $a=a_{0}=a_{1}$ in the notation of Sect. 2.1. 
Lemma 2.1 Assume (C0)(i),(ii) and (C1) with $C^{3}$ replaced by $C^{1}$, and also (C2)(i)(a). Let $I$ be as in $\mathbf{( C 2 )}$. If there is a point in $\bigcap_{n \geq 0} f^{-n}\left(S^{1} \backslash I\right)$ which is not eventually periodic, then $T_{a, b}$ has a horseshoe for all small $b$.

Proof: Relaxing $c_{1}$ to $\frac{c_{1}}{2}$, (C2)(i)(a) continues to hold (for the same $N_{1}$ ) if $I$ in (C2) is replaced by a slightly smaller neighborhood $\tilde{I}$ of the critical set. This implies there exists $\varepsilon>0$ such that for all $x$ with $x, f x, \cdots, f^{n} x \in S^{1} \backslash I$, there is a small interval $J$ containing $x$ such that $J, f(J), \cdots . f^{n}(J) \subset S^{1} \backslash \tilde{I}$ and $f^{n}(J)=\left[f^{n} x-\varepsilon, f^{n} x+\varepsilon\right]$. Let $N \geq N_{1}$ be such that all intervals having the same $N$-itinerary have lengths $<\varepsilon$. Consider $\left\{x: f^{i} x \in S^{1} \backslash \tilde{I}\right.$ for $0 \leq i \leq N\}$, and let $J_{1}, \cdots, J_{r}$ be the components of this set corresponding to distinct itineraries. Define a transition matrix $A$ on $\{1,2, \cdots, r\}$ by letting $a_{i j}=1$ if and only if $f^{N} J_{i} \supset J_{j}$, and let $\Lambda^{+}=\Lambda_{N, A}^{+}=\left\{x \in S^{1}: \forall j \geq 0, \exists i(j)\right.$ s.t. $f^{N j} x \in J_{i(j)}$ and $a_{i(j) i(j+1)}=$ $1\}$. We claim that

$$
\bigcap_{n \geq 0} f^{-n}\left(S^{1} \backslash I\right) \subset \Lambda^{+} \subset \bigcap_{n \geq 0} f^{-n}\left(S^{1} \backslash \tilde{I}\right)
$$

The first containment above is a consequence of our choice of $\varepsilon$ and $N$ : if $x$ is such that $x, f x, \cdots, f^{N} x \in\left(S^{1} \backslash I\right), x \in J_{i}, f^{N} x \in J_{j}$, then $f^{N} J_{i} \supset\left[f^{N} x-\varepsilon, f^{N} x+\varepsilon\right] \supset J_{j}$. Our assumption on $\cap f^{-n}\left(S^{1} \backslash I\right)$ ensures that $\left.f^{N}\right|_{\Lambda^{+}}$is a nontrivial shift of finite type. From this it follows from a standard argument that for some $k, n,\left.f^{N k}\right|_{\Lambda^{+}}$has an invariant set topologically conjugate to $\left(\Sigma_{n}^{+}, \sigma\right)$, the one-sided full shift on $n$ symbols. The second containment in the displayed expression is obvious; it ensures that $\left.f^{N}\right|_{\Lambda^{+}}$is expanding. The existence of a horseshoe now follows immediately from invariant cones arguments.

\section{$3 \quad$ Periodically-kicked Limit Cycles}

This section contains geometric ideas leading to the proof of Theorem 1.

\subsection{Existence of singular limits}

The setting is as follows. Let $\varphi_{t}$ be a flow on a Riemannian manifold $M$ with a periodic orbit $\gamma$. We assume $\gamma$ is a hyperbolic limit cycle, i.e. if $\Sigma$ is a codimension one disk transversal to $\gamma$ at $x$, and $g: \Sigma \rightarrow \Sigma$ is the return map, then all the eigenvalues of $D g(x)$ have modulus $<1$. We consider a periodically kicked system represented by the iteration of $\varphi_{T} \circ \kappa$ where $\kappa: M \rightarrow M$ is a map and $T$ is a long period of relaxation. We will prove in this subsection that under very mild conditions, singular limits in the sense of Sect. 2.1 exist for $\varphi_{T} \circ \kappa$ as $T \rightarrow \infty$.

First we introduce the relevant geometric objects. Let $V=\left\{x \in M: \varphi_{t}(x) \rightarrow \gamma\right.$ as $t \rightarrow \infty\}$. For each $x \in \gamma$, let $W^{s s}(x)$ denote the strong stable manifold through $x$, i.e.

$$
W^{s s}(x)=\left\{y \in M: d\left(\varphi_{t}(x), \varphi_{t}(y)\right) \rightarrow 0 \text { as } t \rightarrow \infty\right\} .
$$

Then $V$ is foliated by $W^{s s}$-leaves each one of which is an immersed codimension one submanifold meeting $\gamma$ in exactly one point. Let $\pi: V \rightarrow \gamma$ be the projection map obtained by sliding along $W^{s s}$-leaves, i.e. for $y \in V, \pi(y)$ is the unique point $x \in \gamma$ such that $y \in W^{s s}(x)$. 
Next we introduce a family of maps in the spirit of Sect. 2.1. Let $p$ be the period of $\gamma$, and let $b_{n}, n=1,2, \cdots$, be a (any) monotonically decreasing sequence of numbers accumulating at 0 . For $n=1,2, \cdots$ and $a \in[0, p)$, we define

$$
T_{a, b_{n}}=\varphi_{n p+a} \circ \kappa
$$

where $\kappa$ is the "kick".

Proposition 3.1 Let $\varphi: M \times \mathbb{R} \rightarrow M$ be any $C^{4}$ flow with a hyperbolic limit cycle $\gamma$, and let $U$ be a tubular neighborhood of $\gamma$. We assume $\kappa: \bar{U} \rightarrow M$ is a $C^{3}$ embedding with $\kappa(\bar{U}) \subset V$. Then for all large $n, T_{a, b_{n}}(U) \subset U$ and the following are true:

(i) $\left\{T_{a, b_{n}}\right\}$ is of the form specified in the paragraph before (C0) in Sect. 2.1;

(ii) conditions $(\mathbf{C 0})$ and $(\mathbf{C 1})$ in Sect. 2.1 are satisfied; and

(iii) the singular limit $T_{a, 0}$ is of the form

$$
T_{a, 0}=\varphi_{a} \circ \pi \circ \kappa
$$

Proof: First, we argue that pointwise for each $y \in U, T_{a, b_{n}}(y) \rightarrow T_{a, 0}(y)$ as $n \rightarrow \infty$ : For $y \in U$, we have $z:=\kappa(y) \in V$, and by definition of $W^{s s}$,

$$
d\left(\varphi_{t}(z), \varphi_{t}(\pi(z))\right) \rightarrow 0 \quad \text { as } t \rightarrow \infty .
$$

That $\left.\varphi_{n p+a}(\pi(z))\right)=\varphi_{a}(\pi(z))$ follows immediately from the fact the period of the cycle is $p$. Since $\kappa(\bar{U})$ is a compact subset of $V$, the convergence above is, in fact, exponentially fast and uniform for all $y \in \bar{U}$. To prove (C0)(iii), let $J$ be such that $\left|\operatorname{det}\left(D \varphi_{p}(z)\right)\right|=e^{-J}$ for all $z \in \gamma$. The exponential convergence above implies that $\left|\operatorname{det}\left(D T_{a, b_{n}}\right)(z)\right|$ differs from $e^{-J n}$ by at most a constant for all $z \in \bar{U}$. The verification of (C0) is complete.

(C1) requires that we prove a stronger form of convergence than that in the last paragraph. Observe that $(a, z) \mapsto T_{a, b_{n}}(z)$ can be written in the composite form

$$
(a, z) \mapsto(a, \kappa(z)) \mapsto\left(a,\left(\varphi_{p}\right)^{n}(\kappa(z))\right) \mapsto \varphi_{a}\left(\left(\varphi_{p}\right)^{n}(\kappa(z))\right)
$$

Since the first map is $C^{3}$ and the last is $C^{4}$, to prove the asserted $C^{3}$-convergence, it suffices to show that $\left(\varphi_{p}\right)^{n}$ converges in $C^{3}$ to $\pi$ as $n \rightarrow \infty$. Recall from standard stable manifolds theory that for a $C^{4}$ flow, each $W^{s s}(x)$-leaf is locally a $C^{4}$ embedded disk [HPS]. This together with the fact that all the leaves of $W^{s s}$ are $\varphi_{t}$-images of $W^{s s}(x)$ for some $x$ implies that as a foliation, $W^{s s}$ is also $C^{4}$. The required convergence is thus tantamount to the following calculus lemma:

Lemma 3.1 Let $I=(0,1), D=\left\{y \in \mathbb{R}^{n-1}:|y|<1\right\}$, and consider $H: I \times D \rightarrow I \times D$ of the form

$$
H(x, y)=(x, h(x, y))
$$

where (i) $h(x, 0)=0$ for all $x \in I$,

(ii) for all $i,\left|\partial_{y_{i}} h\right| \leq \lambda$ for some $\lambda<1$, and

(iii) $\|H\|_{C^{4}}<K$ for some $K>0$.

Then as $n \rightarrow \infty, H^{n}$ converges in the $C^{3}$-norm to $H_{0}$ where $H_{0}(x, y)=(x, 0)$. 
Proof of Lemma 3.1: For notational simplicity, we give a proof only in two dimensions. Let us write $H^{n}(x, y)=\left(x, h_{n}(x, y)\right)$. Since $\left|h_{n}(x, y)\right| \leq \lambda^{n}$, convergence in $C^{0}$ is assured as explained earlier. Computing recursively, we obtain

$$
\begin{gathered}
\partial_{1} h_{n}(\cdot)=\sum_{k=0}^{n-1} \partial_{2} h\left(H^{n-1} \cdot\right) \cdots \partial_{2} h\left(H^{k+1} \cdot\right) \partial_{1} h\left(H^{k} \cdot\right) \\
\partial_{2} h_{n}(\cdot)=\partial_{2} h\left(H^{n-1} \cdot\right) \partial_{2} h\left(H^{n-2} \cdot\right) \cdots \partial_{2} h(\cdot) .
\end{gathered}
$$

It follows immediately that $\left|\partial_{2} h_{n}\right| \leq \lambda^{n}$. Using also $\left|\partial_{1} h(x, y)\right| \leq K|y|$, we see that each term in $\partial_{1} h_{n}(\cdot)$ is bounded above by $\lambda^{n-k-1} \cdot K \lambda^{k}$, so that $\left|\partial_{1} h_{n}\right| \leq K n \lambda^{n-1}$.

Moving on to second derivative estimates, since $\partial_{i j} h_{n}$ is computed by differentiating each factor in each term of $\partial_{j} h_{n}$, we observe that $\partial_{i j} h_{n}$ has $\leq 2 n^{2}$ terms, and each term has $\leq(n+1)$ factors. Moreover, if $\partial_{2} h\left(H^{k} \cdot\right)$ is differentiated, then our previous estimate of $\left|\partial_{2} h\left(H^{k} \cdot\right)\right| \leq \lambda$ is replaced by one of the following: $\left|\partial_{12} h\left(H^{k} \cdot\right)\right| \leq K,\left|\partial_{22} h\left(H^{k} \cdot\right) \partial_{1} h_{k}(\cdot)\right| \leq$ $K^{2} k \lambda^{k-1}$ ( $K$ in the case $\left.k=0\right)$, or $\left|\partial_{22} h\left(H^{k} \cdot\right) \partial_{2} h_{k}(\cdot)\right| \leq K \lambda^{k}$. If $\partial_{1} h\left(H^{k} \cdot\right)$ is differentiated, then our previous estimate of $\left|\partial_{1} h\left(H^{k} \cdot\right)\right| \leq K \lambda^{k}$ is replaced by $\left|\partial_{11} h\left(H^{k} \cdot\right)\right| \leq K \lambda^{k}$ or estimates identical to those above for $\left|\partial_{12} h\left(H^{k} \cdot\right)\right|$. We conclude that $\left|\partial_{i j} h_{n}\right| \leq 2 n^{2} \cdot$ const $\cdot n^{2} \lambda^{n-2}$.

A similar argument gives $\left|\partial_{i j k} h_{n}\right| \leq$ const $n^{\alpha} \lambda^{n-3}$ for some $\alpha$. (The boundedness of the fourth derivative of $H$ is used to control $\partial_{111} h$.)

\subsection{Creation of strange attractors from limit cycles}

We now use the ideas developed in Sect. 3.1 to prove Theorem 1, which says that when suitably kicked, any hyperbolic limit cycle will exhibit chaotic behavior. See Sect. 1.2 for the precise statement.

Proof of Theorem 1: First we produce an open set $\mathcal{E} \subset \mathrm{Emb}^{3}(U, M)$ consisting of suitable kicks. This step is not necessary, but some readers may find it helpful to first "straighten out" the $W^{s s}$-foliation. More precisely, we may assume, via a $C^{4}$ change of coordinates, that $\gamma=\{y=0\}$ and the $W^{s s}$-manifolds are codimension one planes perpendicular to $\gamma$. That this can be done is explained in Sect. 3.1.

One way to choose $\mathcal{E}$ is to begin by selecting a $C^{3}$ map $\Phi: S^{1} \rightarrow \mathbb{R}$ with nondegenerate critical points. Proposition 2.1 gives an open set of $L$ and $\Psi$ such that $f_{a}(\theta):=\theta+a+$ $L(\Phi(\theta)+\Psi(\theta))$ satisfies Conditions (C2), (C3) and (C5). These choices of $L$ and $\Psi$ give rise to a collection of $f_{0}$, which constitutes an open set $\hat{\mathcal{E}}_{0}$ of $C^{3}$ maps from $S^{1}$ to itself. From $\hat{\mathcal{E}}_{0}$, we construct an open set $\mathcal{E}_{0} \subset \operatorname{Emb}^{3}(\gamma, M)$ consisting of $\kappa_{0}$ such that $\pi \circ \kappa_{0}=f_{0}$. Given $f_{0}$, the existence of $\kappa_{0}$ is trivial in dimensions $>2$ : simply lift the image of $\kappa(\gamma)$ in the "vertical" direction to avoid self-intersections. An argument (which we leave as an exercise for the reader) is needed for 2D: use (i) $f_{0}$ has degree one and (ii) our limit cycle $\gamma$ has an orientable normal bundle. From $\mathcal{E}_{0}$ we construct $\mathcal{E} \subset \operatorname{Emb}^{3}(U, M)$ where $\kappa \in \mathcal{E}$ is obtained by extending $\kappa_{0} \in \mathcal{E}_{0}$ to $U$ in such a way that $\partial_{y_{i}}(\pi \circ \kappa) \neq 0$ for some $i$ at points whose $\theta$-coordinates are near the critical points of $\pi \circ \kappa_{0}$. This completes our construction of $\mathcal{E}$. 
For each $\kappa \in \mathcal{E}$, we now introduce, as in Sect. 3.1, a 2-parameter family of maps from a tubular neighborhood of $\gamma$ to itself, namely $T_{a, b_{n}}=\varphi_{n p+a} \circ \kappa$ where $p$ is the period of the cycle and $a \in[0, p)$. Proposition 3.1 says that this family is of the type considered in Sect. 2.1 , and that $(\mathbf{C 0})$ and $(\mathbf{C 1})$ are valid provided $\kappa(U)$ remains in the basin of attraction of $\gamma$. By design, the singular limit is what we started with in the last paragraph, so (C2), (C3), (C4) and, if we so desire, (C5), are met. Thus [WY3] (the relevant portion of which is summarized in Theorems A and B in Sect. 2.1) applies. ${ }^{4}$

[WY3] tells us that for each sufficiently large $n$, there is a positive Lebesgue measure set $\Delta_{n} \subset[0, p)$ such that for all $a \in \Delta_{n}, T_{a, b_{n}}$ has a strange attractor with the properties in (SA1)-(SA4). Thus the dynamical description in Theorem 1(2) holds for all $T \in\{n p+a$ : $a \in \Delta_{n}, n \geq n_{0}$ for some $\left.n_{0} \in \mathbb{Z}^{+}\right\}$. (There is no relation between " $T$ ", the period of the kicks, and $T_{a, b_{n}}$; we regret the unfortunate notation.)

As for Theorem 1(1), if $L$ in the singular limit is sufficiently large, then Lemma 2.1 says that horseshoes are present for all $a$ provided that $b_{n}$ is sufficiently small, i.e. they are present for all $T \geq$ some $T_{0}$.

\subsection{More on production of chaos: example and discussion}

Sect. 3.2 contains an abstract existence result. We now turn to a more practical question: given an arbitrary limit cycle (the way it is embedded in the ambient manifold), what kinds of kicks will result in chaotic behavior?

Example. A linear model. Consider

$$
\begin{aligned}
\dot{\theta} & =1+\sigma \cdot \mathbf{y} \\
\dot{\mathbf{y}} & =-\mathbf{\Lambda} \mathbf{y}+A \Phi(\theta) \mathbf{v}(\theta) \sum_{n=0}^{\infty} \delta(t-n T)
\end{aligned}
$$

where $\theta \in S^{1}, \mathbf{y} \in \mathbb{R}^{n-1}, \sigma$ is a fixed vector in $\mathbb{R}^{n-1}$, and $\boldsymbol{\Lambda}$ is an $(n-1) \times(n-1)$ matrix all of whose eigenvalues have strictly positive real parts. For simplicity, we assume the kicks are perpendicular to the limit cycle $\{\mathbf{y}=\mathbf{0}\}$, with the amplitude of the kicks at $(\theta, \mathbf{y})$ given by $A \Phi_{0}(\theta)$ and the direction by $\mathbf{v} \in S^{n-2}$. The $W^{s s}$-manifolds here are a family of parallel codimension one planes. A simple computation gives

$$
f_{a}(\theta)=\theta+a+A \Phi(\theta) \sigma^{t} \boldsymbol{\Lambda}^{-1} \mathbf{v}(\theta) .
$$

Observe that the effect of the kick is magnified by $\sigma^{t} \boldsymbol{\Lambda}^{-1} \mathbf{v}$, an amount determined by the competition between shear and rate of contraction in the direction of $\mathbf{v}$. In particular, for $n=2$, where $\sigma, \lambda=\boldsymbol{\Lambda} \in \mathbb{R}$ and $\mathbf{v}=+1$ or -1 , we have the following:

Proposition 3.2 (cf. [WY2], Theorems 2 and 3) In dimension 2, given a $C^{3}$ function $\Phi_{0}$ with nondegenerate critical points, there exists $K_{1}=K_{1}\left(\Phi_{0}\right)$ such that if

$$
\frac{\sigma}{\lambda} A:=\frac{\text { shear }}{\text { contraction }} \cdot \text { amplitude of kick }>K_{1},
$$

\footnotetext{
${ }^{4}$ Orientability of the normal bundle to $\gamma$ is assumed in the setup in [WY1] and [WY3], although it is not essential in the proofs.
} 
then the time-T-map of (4) has a horseshoe, i.e. Property $\mathbf{( H )}$, for all large $T$ and a strange attractor with (SA1)-(SA4) for a positive measure set of $T$.

This follows from a direct application of [WY1] (see Sect. 2.1). For (C2), (C3) and (C5), see Proposition 2.1. (C4) is satisfied since $\partial_{y} T_{a, 0}=\frac{\sigma}{\lambda} \neq 0$.

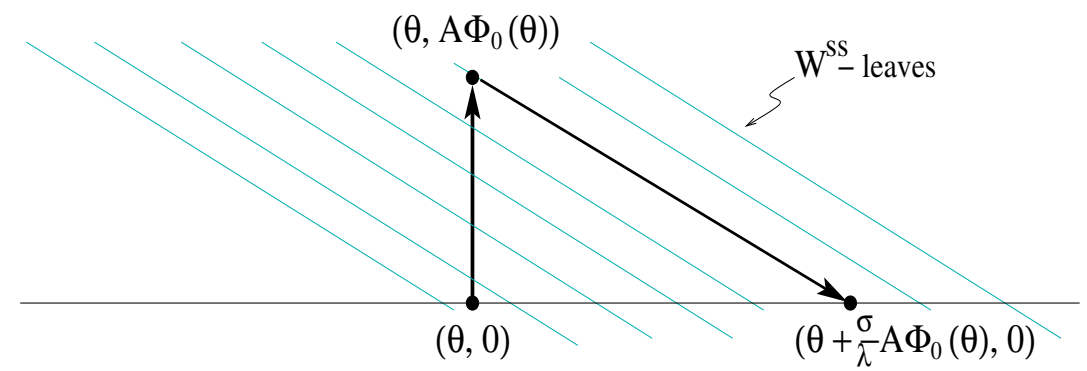

Fig. 2 Geometric view of singular limit for equation (4) in dimension 2

Remark on general situation. Consider now a completely arbitrary limit cycle in any dimension. For the linearized equation, the $W^{s s}$-plane through a point can be computed from the cumulative action as the point moves once around the cycle. When a kick-force is added, its effect is, as in the example above, determined by the angles the $W^{s s}$-planes make with the periodic orbit $\gamma$ and the directions of the kick in relation to the $W^{s s}$-planes. An explicit solution may not be available, but the geometric principles behind it are clear.

The picture rendered by the linearized equation, however, may not be an accurate reflection of that for the nonlinear flow: The smaller the angles between the limit cycle and the $W^{s s}$-leaves, the more prominent is the role played by curvature (or second derivatives). If these angles are small, and if to obtain the singular limit we have to slide a nontrivial distance along (curved) $W^{s s}$-leaves (see Sect. 3.1), then the information given by the linearized equation is even less meaningful. Finally, for our scheme to work, care must be taken to ensure that the kick does not take us outside of the basin of attraction.

From the discussion above, we see that in general, the answer to when chaotic behavior arises depends on fairly detailed information along the limit cycle. In the case of Hopf bifurcations, this information is contained in the first few derivatives at a single point. This together with the frequent occurrence of Hopf bifucations makes it a natural setting for the type of results formulated here.

\section{Proof of Results on Hopf Bifurcations}

For the $2 \mathrm{D}$ result, the situation can be summarized as follows:

1. The dynamical properties in our theorems are derived from the "abstract theory" in [WY1] and [WY3]. In these two papers, we proved that these and other properties are enjoyed by maps satisfying a certain set of conditions. The aim of this section is to prove that these conditions are met by the system defined by equation (3). 
2. The conditions in [WY1] and [WY3], which for the convenience of the reader we have reproduced in Sect. 2.1, are primarily of two types: the first concerns the existence of a singular limit; the second concerns the properties of the maps in the singular limit. These two aspects are discussed separately in the next two paragraphs.

3. The existence of a singular limit has been proved in a much more general setting than is needed here; see Proposition 3.1.

4. Instead of verifying the remaining conditions directly for equation (3), we have identified a class of model 1D maps and proved that if a singular limit belongs in this class, then it has many of the desired properties. See Proposition 2.1.

5. In this section we will prove that the singular limits of equation (3) belong in the class in Propositin 2.1. The delicateness of the situation stems from the fact that we are dealing with a degenerate problem. The degeneracy here is twofold: as $\mu \rightarrow 0$, the limit cycle degenerates to a point, at the same time that it loses its hyperbolicity.

The remainder of this section is organized as follows: The 2D case is treated in Sects. 4.1-4.4. We will focus on proving the presence of strange attractors. The proof for horseshoes follows from a (considerably simpler) version of our arguments here together with Lemma 2.1. The reduction of the $n$-dimensional result to $2 \mathrm{D}$ is carried out in Sect. 4.5.

\subsection{Standardizing coordinates}

We begin by blowing up the neighborhoods of 0 . The purpose of this coordinate change is to standardize the size and position of the limit cycle for all $\mu$. Let $y=r \sqrt{\frac{\alpha}{\mu}}-1$. Then equation (3) in Sect. 1.3 becomes

$$
\begin{aligned}
& \dot{\theta}=\hat{\omega}+\frac{\beta}{\alpha} \mu(y+2) y+\mu^{2} \hat{h}(\theta, y) \\
& \dot{y}=-\mu\left(y^{2}+3 y+2\right) y+\mu^{2} \hat{g}(\theta, y)+(y+1) \Phi(\theta) \sum_{n=0}^{\infty} \delta(t-n T)
\end{aligned}
$$

where $y \in(-1, \infty), \theta \in \mathbb{R} /(2 \pi \mathbb{Z})$, and

$$
\begin{aligned}
\hat{\omega} & =\omega+\left(\gamma+\frac{\beta}{\alpha}\right) \mu, \\
\hat{g}(\theta, y) & =\frac{1}{\alpha^{2}}(y+1)^{5} g\left(\theta, \sqrt{\frac{\mu}{\alpha}}(y+1)\right), \\
\hat{h}(\theta, y) & =\frac{1}{\alpha^{2}}(y+1)^{4} h\left(\theta, \sqrt{\frac{\mu}{\alpha}}(y+1)\right) .
\end{aligned}
$$

Observe that the presence of the $\hat{\omega}$-term in equation (5) prevents us from getting rid of the degeneracy at $\mu=0$ by a simple rescaling of time. 


\subsection{Singular limit in the absence of higher order terms}

In this subsection, we set $\hat{g}=\hat{h}=0$, and consider the flow $\varphi_{t}$ generated by the unforced equation

$$
\begin{aligned}
& \dot{\theta}=\hat{\omega}+\sigma(y) y, \\
& \dot{y}=-\lambda(y) y
\end{aligned}
$$

where

$$
\sigma(y)=\frac{\beta}{\alpha} \mu(y+2), \quad \lambda(y)=\mu\left(y^{2}+3 y+2\right) .
$$

Let $\mu$ be fixed for the rest of this subsection. As explained in Sect. 3.1, the singular limit maps are related to $\lim _{n \rightarrow \infty} \varphi_{t_{n}}$ where $t_{n}=\frac{2 n \pi}{\hat{\omega}}$.

Proposition 4.1 For all small $\mu>0$ and $-1<y<\infty$,

$$
\lim _{n \rightarrow \infty} \varphi_{t_{n}}(\theta, y)=\left(\theta+\frac{\beta}{\alpha} \ln (y+1), \quad 0\right) \text {. }
$$

Let $(\theta(t), y(t))$ denote the solution of $(7)$ with initial conditions $\left(\theta_{0}, y_{0}\right)$. Proposition 4.1 follows immediately from Lemma 4.1 by letting $n \rightarrow \infty$.

\section{Lemma 4.1}

$$
\theta(t)=\theta_{0}+\hat{\omega} t+\frac{\beta}{\alpha} \ln \left(\frac{y_{0}+1}{y(t)+1}\right)
$$

Proof: The reader can verify that this is the solution by direct differentiation. We arrived at the formula above by formally substituting $d s=-\frac{1}{\lambda(y)} d y$ into the integral in

$$
\theta(t)=\theta_{0}+\hat{\omega} t+\int_{0}^{t_{0}} \sigma(y(s)) d s
$$

obtaining

$$
\int_{0}^{t} \sigma(y(s)) y d s=-\int_{y_{0}}^{y(t)} \frac{\sigma(y)}{\lambda(y)} d y=-\frac{\beta}{\alpha} \int_{y_{0}}^{y(t)} \frac{1}{1+y} d y
$$

Let $\pi$ be the projection map in Sect. 3.1. We observe:

(i) $\pi(\theta, y)$, which is the limit in Proposition 4.1, is defined for all $\theta \in S^{1}$ and $y>-1$.

(ii) From the formula for $\pi$, we deduce the following geometric information about the $W^{s s_{-}}$ foliation: its leaves are invariant under translations in the $\theta$-direction; their slopes have the same sign (either positive or negative) everywhere; near $y=0$, the slopes are $\approx-\frac{\beta}{\alpha}$; they tend to $+\infty$ or $-\infty$ as $y \rightarrow \infty$ and to 0 as $y \rightarrow-1$.

(iii) As $\mu \rightarrow 0^{+}, \pi(\theta, y) \rightarrow\left(\theta+\frac{\beta_{0}}{\alpha_{0}} \ln (y+1)\right.$, 0$)$ where $\alpha_{0}$ and $\beta_{0}$ are the values of $\alpha$ and $\beta$ at $\mu=0$. This is strong indication that in spite of the weakening hyperbolicity, the $W^{s s}$-structure remains robust up to $\mu=0$. 


\subsection{Effects of higher order terms}

We continue to consider the unforced equation. Let $\varphi_{t}$ and $\tilde{\varphi}_{t}$ denote respectively the flows with and without higher order terms. When it is useful to identify the parameter $\mu$, we will write $\varphi_{\mu, t}$ and $\tilde{\varphi}_{\mu, t}$. In this subsection and the next, if $X$ is a quantity or object pertaining to $\varphi_{t}$, then the corresponding quantity or object for $\tilde{\varphi}_{t}$ is denoted by $\tilde{X}$.

For each small $\mu>0$, let $\gamma_{\mu}$ denote the limit cycle for $\varphi_{t}$, and call its period $p_{\mu}$. To compare $\varphi_{t}$ and $\tilde{\varphi}_{t}$, we make a time change for $\tilde{\varphi}_{t}$ to synchronize their periods, i.e. to set $\tilde{p}_{\mu}=p_{\mu}$. From the magnitudes of the higher order terms, we see that this time change, which will be assumed for the rest of the proof, is of order $1 \pm \mathcal{O}\left(\mu^{2}\right)$. We also introduce $\iota_{\mu}: \tilde{\gamma}_{\mu} \rightarrow \gamma_{\mu}$ by letting

$$
\iota_{\mu}\left(\tilde{\varphi}_{t}(0,0)\right)=\varphi_{t}\left(0, y_{0}\right)
$$

where $\left(0, y_{0}\right)$ is the point in $\gamma_{\mu}$ whose $\theta$-coordinate is 0 . Next we fix a compact domain of the infinite cylinder $S^{1} \times \mathbb{R}$ in which all the action will take place: let $-1<A_{0}<e^{\min \Phi}-1$ and $A_{1}>e^{\max \Phi}-1$, and let $\mathcal{A}=S^{1} \times\left[A_{0}, A_{1}\right]$. The main result of this subsection is

Proposition 4.2 For $\mu>0$ sufficiently small, $\pi_{\mu}$ is defined on $\mathcal{A}$, and

$$
\left\|\left(\iota_{\mu}^{-1} \circ \pi_{\mu}\right)-\tilde{\pi}_{\mu}\right\|_{C^{3}} \rightarrow 0 \quad \text { as } \mu \rightarrow 0 .
$$

Even though the higher order terms in equation (5) tend to zero as $\mu \rightarrow 0$, this alone is insufficient justification for Proposition 4.2 because as $\mu \rightarrow 0$, the unforced part of equation (5) tends to the totally degenerate, completely integrable system

$$
\dot{\theta}=\omega, \quad \dot{y}=0 .
$$

Let $\boldsymbol{\Phi}_{\mu}=\varphi_{\mu,\left[\frac{1}{\mu p_{\mu}}\right] p_{\mu}}$ and $\tilde{\boldsymbol{\Phi}}_{\mu}=\tilde{\varphi}_{\mu,\left[\frac{1}{\mu p_{\mu}}\right] p_{\mu}}$ where $[x]$ is the greatest integer $\leq x$.

Lemma 4.2 There exists $M$ such that for all small $\mu>0$, the following hold on $\mathcal{A}$ :

(a) $\left\|\boldsymbol{\Phi}_{\mu}\right\|_{C^{4}},\left\|\tilde{\boldsymbol{\Phi}}_{\mu}\right\|_{C^{4}} \leq M$;

(b) $\left\|\boldsymbol{\Phi}_{\mu}-\tilde{\boldsymbol{\Phi}}_{\mu}\right\|_{C^{3}}=\mathcal{O}(\mu)$.

Proof of Proposition 4.2 assuming Lemma 4.2: The task here is to deduce singular limit information, which depends on an infinite number of iterates, from the finite-time information provided by Lemma 4.2 .

Observe that both $\boldsymbol{\Phi}_{\mu}$ and $\tilde{\boldsymbol{\Phi}}_{\mu}$ leave points on their limit cycles fixed, and have uniform "hyperbolic" estimates, i.e. the smaller eigenvalues are uniformly bounded away from 1 and the angles between the stable and neutral directions are uniformly bounded away from 0 .

Let $q_{\mu}=\left(0, y_{0}\right) \in \gamma_{\mu}$. From Lemma 4.2(a) and the uniformness of hyperbolicity, we see that there exists $\hat{\varepsilon}>0$ such that for all small $\mu>0$, the local stable manifolds of $\boldsymbol{\Phi}_{\mu}$ through $q_{\mu}$ and $\tilde{\mathbf{\Phi}}_{\mu}$ through $(0,0)$ are well defined as graphs of $\tau_{\mu}, \tilde{\tau}_{\mu}:[-\hat{\varepsilon}, \hat{\varepsilon}] \rightarrow S^{1}$. We claim that

$$
\left\|\tau_{\mu}-\tilde{\tau}_{\mu}\right\|_{C^{3}} \rightarrow 0 \quad \text { as } \mu \rightarrow 0 .
$$

This is true because from Lemma $4.2(\mathrm{a})$, the set $\mathcal{N}:=\left\{\boldsymbol{\Phi}_{\mu}, \tilde{\boldsymbol{\Phi}}_{\mu} ; \mu \in\left(0, \mu_{0}\right)\right\}$ is bounded in the $C^{4}$-norm, and so it is relatively compact with respect to the $C^{3}$-topology. The mapping $G \rightarrow \tau_{G}$ is continuous with respect to the $C^{3}$ topologies for both $G$ and $\tau_{G}$ (see [HPS]). 
Hence it is uniformly continuous on $\mathcal{N}$. The convergence to 0 of $\left\|\tau_{\mu}-\tilde{\tau}_{\mu}\right\|_{C^{3}}$ now follows from Lemma 4.2(b).

As noted in the last paragraph of Sect. 4.2 , the $W^{s s}$-leaves of $\tilde{\boldsymbol{\Phi}}_{\mu}$ run from top to bottom of the annular region $\mathcal{A}$. Since each of these (long) $W^{s s}$-leaves is contained in the $\left(\tilde{\varphi}_{t}\right)^{-1}$-image of $\operatorname{graph}\left(\tilde{\tau}_{\mu}\right)$ for some $t \leq T_{1}$, it follows that the leaves of the $W^{s s}$-foliation for $\varphi_{t}$ behave similarly and that the two foliations are asymptotically close in $C^{3}$ as $\mu \rightarrow 0$ (meaning there exist diffeomorphisms which converge to the identity in $C^{3}$ carrying the leaves of one to those of the other). By an argument similar to that in the last paragraph, we see also that as $\mu \rightarrow 0, \gamma_{\mu}$ converges in $C^{3}$ to $\tilde{\gamma}_{\mu}=\{y=0\}$, and $\iota_{\mu}$ converges in $C^{3}$ to the identity map. The assertion in Proposition 4.2 follows.

The proof of Lemma 4.2 uses the following elementary fact:

Lemma 4.3 Let $\Omega \in \mathbb{R}^{N}$ be a convex open domain, and let $W$ and $Z$ be $C^{1}$ vector fields on $\Omega$. Suppose that for $t \in\left[0, t_{0}\right], \xi(t), \eta(t) \in \Omega$ are solutions of

$$
\frac{d \xi}{d t}=W(\xi) \quad \text { and } \quad \frac{d \eta}{d t}=Z(\eta)
$$

with $\xi(0)=\eta(0)$. Then for all $t \in\left[0, t_{0}\right]$,

$$
\|\xi(t)-\eta(t)\| \leq \frac{C_{1}}{C_{2}}\left(e^{C_{2} t}-1\right)
$$

where $C_{1}:=\sup _{x \in \Omega}\|W(x)-Z(x)\|$ and $C_{2}:=\sum_{i=1}^{N} \sup _{x \in \Omega}\left\|D Z_{i}(x)\right\|, Z_{i}$ being the component functions of $Z$.

Proof: Writing

$$
\frac{d \xi}{d t}-\frac{d \eta}{d t}=(W(\xi)-Z(\xi))+(Z(\xi)-Z(\eta))
$$

we see that $\|\xi(t)-\eta(t)\| \leq x(t)$ where $x(t)$ satisfies the growth condition $\frac{d x}{d t}=C_{1}+C_{2} x$, $x(0)=0$. The solution of this equation is $x(t)=\frac{C_{1}}{C_{2}}\left(e^{C_{2} t}-1\right)$.

Proof of Lemma 4.2: We rescale time (for both equations) by letting $t^{\prime}=\mu t$ but continue to write $t$ instead of $t^{\prime}$, i.e. $\varphi_{t}$ is now the flow generated by

$$
\begin{aligned}
\dot{\theta} & =\frac{\hat{\omega}}{\mu}+\frac{\beta}{\alpha}(2+y) y+\mu \hat{h}(\theta, y), \\
\dot{y} & =-\left(2+3 y+y^{2}\right) y+\mu \hat{g}(\theta, y) .
\end{aligned}
$$

The analogous time change is made for the equation with no higher order terms.

First we verify that for $t \in[0,1)$ and $\mu \in\left(0, \mu_{0}\right)$, the first four derivatives of $\left.\varphi_{t}\right|_{\mathcal{A}}$ are uniformly bounded. For $\varphi_{t}$ itself, we have $|y(t)-y(0)|=\mathcal{O}(\mu)$. Let $X$ denote the vector field in equation (8). Since

$$
D \varphi_{t}(\cdot)=I+\int_{0}^{t} D X\left(\varphi_{s}(\cdot)\right) D \varphi_{s}(\cdot) d s
$$


and $D X$ and $\varphi_{t}$ are uniformly bounded (the only unbounded term, $\frac{\hat{\omega}}{\mu}$, does not appear), it follows that $D \varphi_{t}$ is uniformly bounded. Bootstrapping our way up, we see that the same result holds for $D^{i}\left(\varphi_{t}\right), i=2,3,4$. A similar argument works for $\tilde{\varphi}_{t}$, proving (a).

Next we wish to apply Lemma 4.3 with $\frac{d \xi}{d t}=W(\xi)$ representing the zeroth through third variational equations of $\varphi_{t}, \frac{d \eta}{d t}=Z(\eta)$ the corresponding equations for $\tilde{\varphi}_{t}$, and $t_{0}=1$. We claim that $C_{1}=\mathcal{O}(\mu)$. This is because all the terms involving $\hat{g}$ or $\hat{h}$ have a copy of $\mu$ in front, and the first time change (made at the beginning of Sect. 4.3) creates a difference of $\mathcal{O}(\mu)$ in the lower degree terms: before the second time change, this difference is $\mathcal{O}\left(\mu^{2}\right)$; it gets multiplied by $\frac{1}{\mu}$ in the second time change. The uniform boundedness of $\left\|D Z_{i}\right\|$ is justified above. The conclusion of Lemma 4.3 is precisely the claim in Lemma 4.2(b).

\subsection{Completing the proof}

We now include back the forcing term in the equation. In the coordinates of Sect. 4.1, we see from Sect. 1.3 that the effect of the kick at time 0 is given by

$$
\kappa(\theta, y):=\left(\theta, y^{+}\right)=\left(\theta, \quad(y+1) e^{\Phi(\theta)}-1\right),
$$

so that starting from $|y|$ small, $\left(\varphi_{t} \circ \kappa\right)(\theta, y) \in \mathcal{A}$ for all $t>0$. We continue to use $\varphi_{\mu, t}$ and $\tilde{\varphi}_{\mu, t}$ to denote the time-t-maps of the flows with and without higher order terms, synchronizing for each $\mu$ the periods of the limit cycles as before.

Proof of Theorems 2: Let $T_{a, 0, \mu}=\lim _{n \rightarrow \infty}\left(\varphi_{\mu, n p_{\mu}+a} \circ \kappa\right)$, and define $\tilde{T}_{a, 0, \mu}, f_{a, \mu}$ and $\tilde{f}_{a, \mu}$ accordingly. As explained in the beginning of this section, it suffices to verify for given $\Phi$ that $T_{a, 0, \mu}$ and $f_{a, \mu}$ satisfy Conditions (C2)-(C5) on a parameter interval $\left(0, \mu_{0}\right)$. Combining the formula for $\kappa$ with Proposition 4.1, we have that for $a \in\left[0, p_{\mu}\right)$,

$$
\tilde{T}_{a, 0, \mu}(\theta, y)=\left(\theta+a+\frac{\beta}{\alpha}(\ln (1+y)+\Phi(\theta)), 0\right)
$$

so that

$$
\tilde{f}_{a, \mu}(\theta)=\theta+a+\frac{\beta}{\alpha} \Phi(\theta)=\theta+a+\frac{\beta}{\alpha} A \Phi_{0}(\theta) .
$$

Identifying $\tilde{\gamma}_{\mu}=S^{1} \times\{0\}$ with $S^{1}$ and using $\iota_{\mu}: \tilde{\gamma}_{\mu} \rightarrow \gamma_{\mu}$ as conjugating map, we obtain $f_{a, \mu}: S^{1} \rightarrow S^{1}$ as

$$
f_{a, \mu}(\theta)=\left(\iota_{\mu}^{-1} \circ \pi_{\mu}\right) \circ\left(\kappa \circ \iota_{\mu}\right)(\theta)+a .
$$

By Proposition 4.2 and the fact that $\kappa \circ \iota_{\mu} \rightarrow \kappa$, we have that $\left\|f_{a, \mu}-\tilde{f}_{a, \mu}\right\|_{C^{3}} \rightarrow 0$ as $\mu \rightarrow 0$. Thus if $\left|\frac{\beta}{\alpha}\right| A$ is sufficiently large, then $f_{a}$ satisfies the conditions in Proposition 2.1. Also, $\partial_{y} T_{a, 0} \approx \partial_{y} \tilde{T}_{a, 0}=\frac{\beta}{\alpha}$. Part (1) of Theorem 2 now follows from Lemma 2.1; part (2) follows from Theorems A and B (see Sect. 2.1).

\subsection{Hopf bifurcations in $n$-dimensions}

The hypotheses and notation are as in Sect. 1.4. First we make precise what is meant by "the unforced equation restricted to $W^{c}$ is in normal form". Let $h: V^{c} \rightarrow V^{s}$ be such that $W^{c}=\operatorname{graph}(h)$, and let $\Pi: \mathbb{R}^{n}=V^{c} \oplus V^{s} \rightarrow V^{c}$ be the projection map. Let $\varphi_{t}$ be the 
given flow on $\mathbb{R}^{n}$, and let $\varphi_{t}^{*}$ be the flow on $V^{c}$ defined by $\varphi_{t}^{*}(z)=\Pi \circ \varphi_{t}(z, h(z))$. Our assumption is that the equation for $\varphi_{t}^{*}$ has the form of equation (2). The twist condition in Theorem 4 is computed from this equation.

Proof of Theorem 3: We carry out in detail the strange attractor part of the proof, leaving the horseshoe part (which is considerably simpler) to the reader as before.

I. Structure of unperturbed flow near 0 . There exist $\mu_{0}>0$ and neighborhoods $R$ of 0 in $\mathbb{R}^{n}$ such that the following hold for all $\mu \in\left[0, \mu_{0}\right)$ :

(i) $\varphi_{t}(R) \subset R$ for all $t>0$.

(ii) Defined everywhere on $R$ is a codimension two $D \varphi_{t}$-invariant strong stable subbundle roughly parallel to $V^{s}$. We denote this subbundle by $E^{s, 2}$ and the invariant manifolds tangent to it by $W^{s, 2}$. By the Invariant Section Theorem, $W^{s, 2}$ as a foliation is $C^{3}$ assuming the flow is $C^{4}$ (see [HPS]). Let $\pi^{2}: R \rightarrow W^{c}$ be projection by sliding along $W^{s, 2}$-leaves.

(iii) $\varphi_{t}$ has a limit cycle $\gamma$ contained in $W^{c}$. Through each point in $\gamma$ passes a codimension one strong stable manifold which we denote by $W^{s, 1}$. (These are the $W^{s s}$-manifolds in previous sections.) Let $\pi^{1}$ be the projection onto $\gamma$ by sliding along $W^{s, 1}$-leaves. Note that wherever $W^{s, 1}$ is defined, its leaves contain those of $W^{s, 2}$.

II. Reduction of problem. For each $\mu$, let $T_{a, b_{n}}$ be defined as in Sect. 3.1. We are guaranteed for general reasons the existence of a well defined singular limit $T_{a, 0}$. The problem is reduced to proving (C2)-(C5) for this singular limit.

Since $T_{a, 0}$ alone matters, and (C4) requires only that we guarantee a nonzero derivative for $T_{a, 0}$ in some direction normal to $\gamma$, while (C2), (C3) and (C5) pertain to the restriction of $T_{a, 0}$ to $\gamma$, it may be sufficient to restrict the domain of $T_{a, 0}$ to $W^{c}$, that is to say, the problem is reduced to one in $2 \mathrm{D}$, involving the flow $\left.\varphi_{t}\right|_{W^{c}}$ and kick $\hat{\kappa}:=\left.\pi^{2} \circ \kappa\right|_{W^{c}}$.

Now the hypotheses of Theorem 3 are on the kick-system $\left(\varphi_{t}^{*},\left.\kappa\right|_{V^{c}}\right)$ (note that $\kappa$ leaves $V^{c}$ fixed). To make use of this information, we project the kick-system $\left(\left.\varphi_{t}\right|_{W^{c}}, \hat{\kappa}\right)$ to $V^{c}$, resulting in $\left(\varphi_{t}^{*}, \kappa^{*}\right)$ where the kick map $\kappa^{*}: V^{c} \rightarrow V^{c}$ is given by $\kappa^{*}(z)=\Pi \circ \pi^{2} \circ \kappa(z, h(z))$.

The problem is thus reduced to comparing the two systems $\left(\varphi_{t}^{*},\left.\kappa\right|_{V^{c}}\right)$ and $\left(\varphi_{t}^{*}, \kappa^{*}\right)$, the objective being to deduce singular limit information about the second from that of the first.

III. Magnified coordinates. The proof of Theorem 2 is carried out in blown-up coordinates. Accordingly, we consider an $\mathcal{O}(\sqrt{\mu})$-neighborhood of 0 in $\mathbb{R}^{n}$ and magnify coordinates (in all directions) by a factor $\sim \frac{1}{\sqrt{\mu}}$, obtaining for $\varphi_{t}^{*}$ a limit cycle of radius $\approx \mathcal{O}(1)$. Since magnification decreases higher derivatives, $W^{c}$ and the $W^{s, 2}$-leaves are increasingly "straight" as $\mu \rightarrow 0$. More precisely, in coordinates magnified by $\sim \frac{1}{\sqrt{\mu}},\|h\|_{C^{3}}$ and $\left\|\Pi \circ \pi^{2}-\Pi\right\|_{C^{3}}$ tend to 0 as $\mu \rightarrow 0$.

As for $\kappa$, since this map is scale invariant, meaning $\kappa(r z)=r \kappa(z), r>0$, we have, in magnified coordinates, $\left\|\left.\kappa\right|_{A}\right\|_{C^{3}}=\mathcal{O}(1)$ where $A=\left\{\frac{3}{4}<|z|<\frac{5}{4}\right\}$. (It is necessary to bound the domain away from 0 because $\kappa$ is not differentiable at 0 .)

IV. Comparison of $\left(\varphi_{t}^{*},\left.\kappa\right|_{V^{c}}\right)$ and $\left(\varphi_{t}^{*}, \kappa^{*}\right)$ in magnified coordinates. We write $\kappa^{*}-\left.\kappa\right|_{V^{c}}$ as

$$
\begin{aligned}
& \Pi \circ \pi^{2} \circ \kappa(z, h(z))-\kappa(z, 0) \\
= & {\left[\left(\Pi \circ \pi^{2}-\Pi\right) \circ \kappa(z, h(z))\right]+[\Pi \circ(\kappa(z, h(z))-\kappa(z, 0))] . }
\end{aligned}
$$


From the fact that $\|h\|_{C^{3}}=o(1),\|\kappa\|_{C^{3}}=\mathcal{O}(1)$ in the relevant region, and $\left\|\Pi \circ \pi^{2}-\Pi\right\|_{C^{3}}=$ $o(1)$, we see that $\left\|\kappa^{*}-\left.\kappa\right|_{V^{c}}\right\|_{C^{3}}=\mathcal{O}(1)$ and $\left\|\kappa^{*}-\left.\kappa\right|_{V^{c}}\right\|_{C^{2}}=o(1)$ as $\mu \rightarrow 0$. It follows that in these coordinates, the singular limit maps $T_{a, 0}$ corresponding to the two kick-systems are also $C^{3}$-bounded and $C^{2}$-near each other.

By our assumptions on $\left(\varphi_{t}^{*},\left.\kappa\right|_{V^{c}}\right)$ and from our proof in the 2D case, we know that for this system $f_{a}$ is in the model class considered in Sect. 2.2. By Proposition 2.1, this model class is robust under the type of perturbations above, and maps in this class satisfy (C2), (C3) and (C5). Similarly, information for (C4) is passed from one system to the other.

The desired result for the $n$-dimensional system is proved.

Acknowledgement: The authors thank K. Lu and M. Shub for helpful conversations.

\section{References}

[BC] M. Benedicks and L. Carleson, The dynamics of the Hénon map, Ann. Math. 133 (1991), 73-169.

[CL] M. L. Cartwright and J. E. Littlewood, On nonlinear differential equations of the second order, J. London Math. Soc. 20 (1945), 180-189.

[GH] J. Guckenheimer and P. Holmes, Nonlinear oscillators, dynamical systems and bifurcations of vector fields, Springer-Verlag, Appl. Math. Sciences 42 (1983).

[HPS] M. Hirsch, C. Pugh and M. Shub, Invariant Manifolds, Lecture Notes in Math., Springer Verlag, 583 (1977).

[H] E. Hopf, Abzweigung einer periodischen lösung von einer station-ären lösung eines differentialsystems, Ber. Verh. Sächs, Acad. Wiss. Leipzig Math. Phys., 94 (1942), 1-22.

[J] M. Jakobson, Absolutely continues invariant measures for one-parameter families of onedimensional maps, Comm. Math. Phys. 81 (1981), 39-88.

[K] A. Katok, Lyapunov exponents, entropy and periodic points for diffeomorphisms, Publ. IHES, 51 (1980), 137-173.

[Li] M. Levi, Qualitative analysis of periodically forced relaxation oscillations, Mem. AMS, 214 (1981), 1-147.

[Ln] N. Levinson, A second order differential equation with singular solutions, Ann. Math., 50 No. 1 (1949), 127-153.

[L] M. Lyubich, Almost every real quadratic map is either regular or stochastic, Ann. Math. (2001)

[MM] J. Marsden and M. McCracken, The Hopf bifurcation and its applications, Springer-Verlag, Appl. Math. Sc. 19 (1976).

[MV] L. Mora and M. Viana, Abundance of strange attractors, Acta. Math. 171 (1993), 1-71.

[N] S. Newhouse, "Lectures in Dynamical Systems", in CIME Lectures, Bressanone, Italy, June 1978, Birkhäuser (1980), 1-114

[NRT] S. Newhouse, D. Ruelle and F. Takens, Occurrence of strange Axiom A attractors near quasiperiodic flows on $T^{m}, n \geq 3$, Commun. Math. Phys. 64 (1978), 35-40.

[RT] D. Ruelle and F. Takens, On the nature of turbulence, Commun. Math. Phys. 20 (1971), 167-192. 
[S] S. Smale, Differentiable dynamical systems, Bull. AMS 73, (1967), 717-817.

[WY1] Q. Wang and L.-S. Young, Strange attractors with one direction of instability, Commun. Math. Phys. 218 (2001), 1-97.

[WY2] Q. Wang and L.-S. Young, From invariant curves to strange attractors, Commun. Math. Phys. 225 (2002), 275-304.

[WY3] Q. Wang and L.-S. Young, Strange attractors with one direction of instability in ndimensional spaces, 2002 preprint.

[Y] L.-S. Young, What are SRB measures, and which dynamical systems have them? to appear in a volume in honor of D. Ruelle and Ya. Sinai on their 65th birthdays, J Stat. Phys. (2002).

[Z] G. Zaslavsky, The simplest case of a strange attractor, Physics Letters, 69A, number 3 (1978) $145-147$. 